QMUL-PH-05-1

\title{
D-brane dynamics near compactified NS5-branes
}

\author{
Steven Thomas ${ }^{1}$, John Ward ${ }^{2}$ \\ Department of Physics \\ Queen Mary, University of London \\ Mile End Road \\ London, E1 4NS U.K.
}

\begin{abstract}
We examine the dynamics of a $D p$-brane in the background of $k$ coincident, parallel NS5branes which have had one of their common transverse directions compactified. We find that for small energy, bound orbits can exist at sufficiently large distances where there will be no stringy effects. The orbits are dependent upon the energy density, angular momentum and electric field. The analysis breaks down at radial distances comparable with the compactification radius and we must resort to using a modified form of the harmonic function in this region.
\end{abstract}

\footnotetext{
${ }^{1}$ e-mail: s.thomas@qmul.ac.uk

${ }^{2}$ e-mail: j.ward@qmul.ac.uk
} 


\section{Introduction.}

There has been much recent study of time dependence in string theory, particularly involving supergravity backgrounds [2], [5], [8], [9], [11], [23]. The basic idea has been to probe a given background geometry through the introduction of a single $D p$-brane, whose dynamics can be studied using an effective Born Infeld action. Some of this work has involved probing $D$-brane backgrounds [7], [20], [5], [6], whilst other work has focused on the NS5-brane backgrounds (see [2], [18], [19], [22], [24], [25])

The NS5-brane case is of interest since this particular configuration breaks all the supersymmetry of type II string theory and the BPS probe brane will be unstable. This instability can be interpreted in terms of a rolling tachyon [2], [13], [14], and so study of this system provides useful information about tachyon condensation. In addition, since the NS5-brane is much heavier than the $D p$-brane, the source branes will curve the space around them creating an infinite throat. As one travels down the throat, one finds that the string coupling increases, thus we find that we are moving from the perturbative to the non-perturbative realm.

Since we are using perturbative techniques, we expect that our solution will break down as we approach the bottom of the throat. However [2] found that there were certain values of energy for which the solution held for some time. Another point to consider is the energy emission associated with the $D p$ brane. As it moves in the throat, the probe emits closed strings which carry away energy and the R-R charge. It was found in [9], [23] that the classical analysis is only valid for $5>p>2$, since the energy loss is not divergent in this instance. This will be assumed throughout.

Recent work [8], [19], has emphasised that probing the compact dimension of the $\mathbf{R}^{\mathbf{3}} \times \mathbf{S}^{\mathbf{1}}$ geometry is far better understood in terms of tachyon condensation. However, we expect that the effective DBI action is more useful for probing $\mathbf{R}^{3}$. It is important to understand the motion of branes in time dependent compactified backgrounds, since there may be implications for brane world cosmology. [3], [5], [16].

The purpose of this work will be to attempt to address some of the questions raised in [2] regarding bound orbits and critical angular momenta. We will begin with a review of the background solutions and the effective action for a BPS probe $D p$-brane. We will then briefly look at the behaviour of the 'tachyon' potential before moving on to discuss the dynamics of the probe brane, both with and without angular momentum, where we will try to explicitly solve the equations of motion. We will also briefly investigate what happens when the compact dimension is not infinitesimally small, and its effect on the probe brane. We conclude with some brief remarks and suggestions for future work. 


\section{Effective action and background solution.}

We want to investigate the dynamics of a BPS Dp-brane in the background of $k N S 5$ branes which are located at the origin of the transverse space-time $\mathbf{S}^{1} \times \mathbf{R}^{3}$. To this end, we must first remind ourselves of the CHS solutions for the metric, dilaton and $N S-3$ form, which are given by [1],

$$
\begin{aligned}
d s^{2} & =\eta_{\mu \nu} d x^{\mu} d x^{\nu}+H\left(x^{m}\right) \delta_{m n} d x^{m} d x^{n} \\
e^{2\left(\phi-\phi_{0}\right)} & =H\left(x^{m}\right) \\
H_{m n p} & =-\epsilon_{m n p}^{q} \partial_{q} \phi
\end{aligned}
$$

As usual $H_{m n p}$ is the field strength for the $N S$ B-field, whilst $H\left(x^{m}\right)$ is the harmonic function describing the location of the $k$ fivebranes in the transverse space. Generically, the harmonic function can be written as

$$
H=1+\sum_{i=1}^{k} \frac{l_{s}^{2}}{\left|\mathbf{x}-\mathbf{x}_{i}\right|^{2}},
$$

which, for coincident branes, becomes

$$
H=1+\frac{k l_{s}^{2}}{r^{2}}
$$

where $r=\sqrt{x^{m} x_{m}}$ represents the radial distance from the source branes in the transverse space $\mathbf{R}^{4}$. We wish to consider a new background for the fivebranes, which we can create by compactifying one of the transverse directions into a circle of radius $R_{c}$ (see [4], [15] for example). We are free to arbitrarily choose a direction to compactify since there exists an $S O(4)$ symmetry in the transverse space. If we parameterise our chosen direction by $y$ then the harmonic function can be written:

$$
H=1+k l_{s}^{2} \sum_{n=-\infty}^{\infty} \frac{1}{r^{2}+\left(y-2 \pi R_{c} n\right)^{2}}
$$

If we now assume that the compactification radius is very small, then we immediately see that this summation changes only very slowly with $n$ and can be approximated by an integral

$$
H=1+k l_{s}^{2} \int \frac{d n}{r^{2}+\left(y-2 \pi R_{c} n\right)^{2}} .
$$

Performing a change of variables $r u=2 \pi R_{c} n-y$ and integrating over $u$ gives us the modified harmonic function,

$$
H=1+\frac{\bar{k}}{r}
$$

where for simplicity we have defined $\bar{k}=k l_{s}^{2} / 2 R_{c}$. This has immediate consequences for the validity of the solutions, since we must now ensure that the following constraints are satisfied for the supergravity solution to hold.

$$
r>>R_{c} \quad k l_{s}^{2}>>r R_{c} .
$$


If the radial distance $r$ approaches $R_{c}$ then this solution will no longer be valid. We can still find an expression in this instance, since although the denominator in the integral will no longer be a smoothly changing function, we can still use the summation equation (2.4). This will yield a new harmonic function valid deep in the CHS throat which will match onto the $1 / r$ function at some distance. We will refer to this 'deep throat' approximation later in the paper. For now, we will be concerned with the geometry parameterised by (2.6).

Into this background we wish to introduce a BPS Dp-brane, which is extended in the $x^{1} \ldots x^{p}$ directions and parallel to the NS5 branes. As is usual in this situation, we label the world-volume coordinates by $\zeta^{\mu}$ and go to static gauge where $\zeta^{\mu}=x^{\mu}$. The embedding of the D-brane in the transverse space gives rise to scalar fields on its worldvolume, labelled by $X^{6}\left(\zeta^{\mu}\right) \ldots X^{9}\left(\zeta^{\mu}\right)$, and consequently we find that the brane dynamics may be effectively described by the Born-Infeld action:

$$
S=-\tau_{p} \int d^{p+1} \zeta e^{-\left(\phi-\phi_{0}\right)} \sqrt{-\operatorname{det}\left(G_{\mu \nu}+B_{\mu \nu}+2 \pi l_{s}^{2} F_{\mu \nu}\right)}
$$

where $G_{\mu \nu}$ and $B_{\mu \nu}$ are the pullbacks of the metric and the B-field to the branes world volume, whilst $F_{\mu \nu}$ is the $U(1)$ gauge field and $l_{s}$ is the string length. In this note we are interested in the case where the only excited field on the brane is $r\left(\zeta^{\mu}\right)$, and so the $B$ field couplings vanish. Furthermore, we will only be interested in homogeneous solutions where the scalar fields are explicitly time dependent only. In this case the induced metric becomes

$$
G_{\mu \nu}=\eta_{\mu \nu}+\delta_{\mu}^{0} \delta_{\nu}^{0} \dot{X}^{m} \dot{X}^{m} H\left(x^{n}\right) .
$$

Inserting this into (2.8), and also including a non zero, constant electric field on the brane gives the resultant D-brane action:

$$
S=-\tau_{p} \int d^{p+1} \zeta \sqrt{H^{-1}\left(1-\lambda \varepsilon^{2}\right)-\dot{X}^{m} \dot{X}^{m}}
$$

where $\lambda=2 \pi l_{s}^{2}$, and $\varepsilon$ is the constant electric field strength on the world-volume. Note that the DBI action can be used here since it accurately describes the classical open string motion. It will not however, account for the radiative corrections due to closed string emission [9].

\section{Tachyon map.}

Tachyons in open string models are described by a Born Infeld action, which is given by $[17]$

$$
S=-\int d^{p+1} \zeta V(T) \sqrt{1+\partial_{\mu} T \partial_{\nu} T}
$$


where $V(T)$ is the tachyon potential. Upon comparison with (2.10) we find that setting the electric field to zero there is a map from one to the other, provided we define a tachyon field

$$
\frac{d T}{d r}=\sqrt{H(r)}=\sqrt{1+\frac{\bar{k}}{r}}
$$

And consequently we know that the potential can be written as

$$
V(T)=\frac{\tau_{p}}{\sqrt{H(r)}}
$$

With this definition we find

$$
T(r)=\sqrt{r^{2}+\bar{k} r}+\frac{1}{2} \bar{k} \ln \left(\frac{1}{2} \bar{k}+r+\sqrt{r^{2}+\bar{k} r}\right) .
$$

Ideally we want to invert this equation to obtain an exact expression for the tachyon potential, however this is not always possible and so we must study the effective potential behaviour in its asymptotic limits. To leading order, we find that

$$
\begin{gathered}
T\left(r \rightarrow R_{c}\right) \propto \frac{\bar{k}}{2} \ln \left(\frac{\bar{k}}{2}\right) \\
T(r \rightarrow \infty) \propto r .
\end{gathered}
$$

We find that as $r \rightarrow R_{c}, T \rightarrow$ constant dependent upon the number of branes and the compactification radius. In the other limit we see that as $r \rightarrow \infty, T \rightarrow \infty$ as expected. Using this and also (3.3) we find the effective behaviour of the tachyon potential.

$$
\frac{V(T)}{\tau_{p}} \propto 1-\frac{\bar{k}}{2 T} \quad T \rightarrow \infty .
$$

Thus as $T \rightarrow \infty$ we see that the potential changes as $1 / T \propto 1 / r$ as expected from considering the gravitational attraction. This agrees nicely with the solution in the uncompactified case, where it was found that

$$
\frac{V(T)}{\tau_{p}} \propto\left\{\begin{array}{cc}
e^{T / \sqrt{k l_{s}^{2}}} & T \rightarrow-\infty \\
1-\frac{k l_{s}^{2}}{2 T^{2}} & T \rightarrow \infty
\end{array}\right.
$$

Looking at the $T \rightarrow \infty$ case, we find that if $R_{c}=T$ then the solution in (3.7 is mapped to the solution in (3.8). This is because letting $T$ be large effectively 'blows up' the compact dimension and we therefore recover our $S O(4)$ symmetry in the transverse space. Thus we should expect to recover the Kutasov result [2]. The behaviour of the tachyon field as $r \rightarrow R_{c}$ is logarithmic, which suggests that the potential would have some 
form of exponential behaviour ( [2], [14]), albeit different from that in the uncompactified case. Geometrically we argue that the tachyon field is related to the radial distance between the $D$-brane probe and the fivebranes, and therefore by considering the brane dynamics we are learning about the behaviour of the tachyon.

\section{Probe brane dynamics.}

We are now in a position to consider the dynamics of the probe brane in this compactified background. Referring back to (2.10) we note that we can use the $S O(3)$ symmetry to rotate the solution to the $x_{8}-x_{9}$ plane by switching to polar coordinates. Namely, $x_{8}=R \cos \theta$ and $x_{9}=R \sin \theta$. The action now reads;

$$
S=-\tau_{p} \int d^{p+1} \zeta \sqrt{H^{-1}\left(1-\lambda \varepsilon^{2}\right)-\dot{R}^{2}-R^{2} \dot{\theta}^{2}}
$$

and we can construct the canonical momenta and canonical energy as usual. Note that now, $R$, is the radial distance in this plane and should not be confused with the compactification radius $R_{c}$.

$$
\begin{gathered}
\tilde{\Pi}=\frac{\Pi}{m}=\frac{\dot{R}}{\sqrt{H^{-1}\left(1-\lambda \varepsilon^{2}\right)-\dot{R}^{2}-R^{2} \dot{\theta}^{2}}} \\
\tilde{L}=\frac{L}{m}=\frac{R^{2} \dot{\theta}}{\sqrt{H^{-1}\left(1-\lambda \varepsilon^{2}\right)-\dot{R}^{2}-R^{2} \dot{\theta}^{2}}} \\
\tilde{E}=\frac{1}{H \sqrt{H^{-1}\left(1-\lambda \varepsilon^{2}\right)-\dot{R}^{2}-R^{2} \dot{\theta}^{2}}} .
\end{gathered}
$$

In deriving these expressions we have used the fact that $m=\tau_{p} \int d^{p} \zeta$ represents the effective 'mass' of the brane.

In order to solve the equation of motion for $R$ in terms of fixed energy and angular

momentum densities, we need to solve the angular momentum term for $\dot{\theta}$ and substitute the solution into the radial momentum equation. The resultant expression is

$$
\dot{R}^{2}=\frac{\left(1-\lambda \varepsilon^{2}\right)}{H}-\frac{1}{E^{2} H^{2}}\left(1+\frac{\tilde{L}^{2}}{R^{2}}\right)
$$

Since this expression is non negative, it places constraints upon the strength of the electric field, which can be seen by simply substituting in the expression for the harmonic function. Following recent work on similar problems, it is convenient to define the effective potential to be minus the radial equation of motion. 
Using our expression for the probe brane action we can calculate the energy momentum tensor associated with it. Considering only the time dependent case we find the non zero components of the tensor are:

$$
\begin{gathered}
T_{00}=\frac{\tau_{p}}{H \sqrt{H^{-1}\left(1-\lambda \varepsilon^{2}\right)-\dot{R}^{2}-R^{2} \dot{\theta}^{2}}} \\
T_{i j}=-\tau_{p} \delta_{i j} \sqrt{H^{-1}\left(1-\lambda \varepsilon^{2}\right)-\dot{R}^{2}-R^{2} \dot{\theta}^{2}} .
\end{gathered}
$$

As we can see, the time component of the tensor is associated with the energy density on the brane. The spatial component can be rewritten in terms of this density as;

$$
T_{i j}=\frac{-\delta_{i j} \tau_{p}}{H \tilde{E}}
$$

For small $R$, which we are calling the near horizon geometry, this can be written as

$$
T_{i j} \propto \frac{-\delta_{i j} \tau_{p} R}{\bar{k} \tilde{E}}
$$

which tends to zero linearly with distance, in contrast to the original case which vanished quadratically. It must be remembered that this is only valid for $R>>R_{c}$, and so we cannot map this solution to the Kutasov one [2] in this region.

\subsection{Radial motion in the near horizon limit.}

In this section we consider purely radial motion of the probe brane, and so we drop the angular momentum terms. The constraint equation reduces to:

$$
\frac{\bar{k}\left(1-\lambda \varepsilon^{2}\right)}{R}-\lambda \varepsilon^{2} \geq \frac{1}{\tilde{E}^{2}}-1
$$

This tells us that $\lambda \varepsilon^{2}$ must be bounded above by unity, which is automatically satisfied since we have considered the field to be a small perturbation in order to derive the $D$-brane action. The effective potential in this instance reduces to,

$$
V_{\text {eff }}=\frac{1}{\tilde{E}^{2} H^{2}}-\frac{\left(1-\lambda \varepsilon^{2}\right)}{H}
$$

which is plotted in fig 1 . It shows that the potential is attractive for small energy density, becoming more repulsive as the energy increases. We also see there are minima in the potential at large radial distances, which could give rise to bound orbits. The effect of the increasing energy is to move the minimum to larger distances from the fivebranes.

We can extract useful information about the behaviour of the probe by considering the limit of small and large $R$. As $R \rightarrow 0$, we neglect the factor of unity in the harmonic 
function since the probe is located inside the throat of the geometry. As the probe travels down the throat, is moves into a region of stronger coupling and so we expect our perturbative solutions to break down. But we already know that this is the case, since at very small $R$ the probe will 'feel' the effect of the compactification. The potential in this region can be approximated by

$$
V_{\text {eff }} \propto \frac{R^{2}}{\tilde{E}^{2} \bar{k}^{2}}-\frac{R\left(1-\lambda \varepsilon^{2}\right)}{\bar{k}}
$$

which can be seen to tend to zero as the radial distance decreases for arbitrary energy density. We also note that the potential is identically zero at the radial distance $R=$ $\tilde{E}^{2} \bar{k}\left(1-\lambda \varepsilon^{2}\right)$. This implies that there is some turning point in the potential for a certain range of values of energy and the electric field. It also implies that due to the supergravity constraints (2.7) we must have $\tilde{E}^{2}\left(1-\lambda \varepsilon^{2}\right)<<1$.

In this region, we can solve the equation of motion explicitly. Upon integration we find that

$$
R(t)=\frac{\left(1+\tan (y)^{2} \pm \sqrt{\left.\tan (y)^{2}+\tan (y)^{4}\right)}\right) \bar{k} \tilde{E}^{2}\left(1-\lambda \varepsilon^{2}\right)}{2\left(1+\tan (y)^{2}\right)}
$$

where we have defined $y=t /(\tilde{E} \bar{k})$.

We note that the solution above with - sign corresponds to an inwardly moving probe with initial (ie maximum ) distance from the $N S 5$-branes, given by $R=\bar{k} \tilde{E}^{2}\left(1-\lambda \varepsilon^{2}\right) / 2$. Temporarily setting the electric field to zero we see this implies that the smaller we make $R_{c}$ then the further away the probe will be. By turning on the electric field we see that this has an affect on the maximum distance. In fact we note that this distance decreases as we increase the strength of the electric field. Thus for a fixed value of $R_{c}$ we see that the increasing field strength puts the probe brane closer to the fivebranes.

Another point worth noting is that since tan functions are defined such that the argument lies between $-\pi / 2$ and $\pi / 2$, for a fixed energy density, there are strict constraints on the time evolution of our solution. This is to be expected since we know that the probe must be deep in the fivebrane throat. What the solution tells us though, is that as $y \rightarrow \pi / 2, R(t) \rightarrow 0$. This means that the probe will reach the source branes in a finite time as measured from the fivebranes. This is interesting since it contrasts with the uncompactified case, where that particular solution suggested that it would take an infinite amount of time as measured by the fivebrane, but only a finite time when measured using the proper time on the $D$-brane.

Even though the minus sign solution corresponds to probe motion toward the fivebranes we know that in the $R \rightarrow 0$ limit the theory will break down since the compact dimension will in essence 'decompactify'. Furthermore, there will be strong stringy effects to take into account, as discussed by [2], [9].

For the solution with positive sign in (4.13), corresponding to motion away from 
the fivebranes, we see that the probe potentially starts at some minimum distance. With time evolution it moves away to some maximum distance before returning to its original position. However at this point, it would be expected to match onto the in-falling solution. The maximum distance for the outgoing probe is given by

$$
R_{\max }=\bar{k} \tilde{E}^{2}\left(1-\lambda \varepsilon^{2}\right),
$$

which is also the place where the effective potential vanishes. The difficulty with this solution is that it is unphysical. A probe brane (with electric field) moving into the throat from Minkowski space will either have too much energy and simply head toward the fivebranes, or will oscillate around the minimum of the gravitational potential.

In the large $R$ approximation we know that the background spacetime is flat Minkowski, and so the effective potential goes as

$$
V_{\text {eff }} \propto \frac{1}{\tilde{E}^{2}}-\left(1-\lambda \varepsilon^{2}\right)
$$

which is large and positive for $\tilde{E}<<1$, and small and negative for $\tilde{E}>>1$. Thus we see that the potential is attractive for small energy density and repulsive for large energy density. Combining this with what we know from the throat approximation, we note that for $\tilde{E}<1$ the potential will lead to a bound state since it has a crossing point at a non zero value of $R$. In terms of the tachyon description we see that the $D$-brane will be unstable and will roll down the potential. For $\tilde{E}>1$ the potential is repulsive and will not lead to the formation of a bound state, which is to be expected since we know that this will violate the supergravity constraints. The effect of an increased electric field strength is to shift the potential, reducing the depth of the minimum and bringing it closer to the fivebranes. This is shown in figure 2 .

The explicit solution of the equation of motion in this region is given by

$$
R(t)=t \sqrt{\left(1-\lambda \varepsilon^{2}\right)-1 / \tilde{E}^{2}}
$$

which shows that the probe brane approaches the throat linearly with time, as measured by an observer on the fivebranes.

\subsection{Combined radial and angular motion.}

With the inclusion of the angular momentum density, we expect to see slightly altered dynamics. We again investigate the potential in the two limits as in the previous section. Firstly, for motion in the throat the effective potential is;

$$
V_{\text {eff }} \propto \frac{R^{2}}{\tilde{E}^{2} \bar{k}^{2}}\left(1+\frac{\tilde{L}^{2}}{R^{2}}\right)-\frac{R\left(1-\lambda \varepsilon^{2}\right)}{\bar{k}}
$$


which does not vanish as $R \rightarrow 0$, in fact it tends to a constant dependent upon the energy density and the angular momentum density. In all cases we find that the potential is repulsive in the throat, independent of the size of the energy density. (4.16) also provides us with another constraint on the allowed values of the energy density and angular momentum. It turns out that they must satisfy

$$
\frac{1}{\tilde{E}^{2}\left(1-\lambda \varepsilon^{2}\right)}\left(1+\frac{\tilde{L}^{2}}{R^{2}}\right)>>1
$$

The solution to the equation of motion in the throat is given by

$$
R(t)=\frac{\tilde{E}^{2} \bar{k}\left(1-\lambda \varepsilon^{2}\right)\left(1+\tan (y)^{2}\right) \pm \sqrt{\tilde{E}^{4} \bar{k}^{2}\left(1-\lambda \varepsilon^{2}\right) B-4 \tilde{L}^{2} B}}{2\left(1+\tan (y)^{2}\right)}
$$

Where $y$ is defined as before, and $B=\tan (y)^{2}+\tan (y)^{4}$. Once again we see that at $t=0$, the probe is located at $\bar{k} \tilde{E}^{2}\left(1-\lambda \varepsilon^{2}\right) / 2$. Choosing the minus sign again corresponds to inward motion. We see that the solution describes some kind of oscillatory motion, but now there is the additional angular momentum term to consider.

There appears to be a critical value for the angular momentum emerging from this solution, and the vanishing of the potential.

$$
\tilde{L} \leq \frac{\tilde{E}^{2} \bar{k} \sqrt{1-\lambda \varepsilon^{2}}}{2}
$$

If the angular momentum satisfies this condition, then the solution is valid. However in the limit that the LHS is equal to the right hand side, then the square root term in (4.18) vanishes. This means that the whole equation for $R$ is independent of time, and so the probe brane will be at a constant distance from the fivebranes. Physically we can think of it as rotating rapidly around the throat. The position of the probe in this case is dependent upon the size of $R_{c}$ and the electric field strength. This suggests that if $\tilde{E}<1$ then the angular momentum term is vanishingly small, and can really only be defined for the $\tilde{E}>1$ case. If the constraint is violated then we pick up an imaginary term in the equation of motion. If we look at the vanishing of the potential, we find the solutions are

$$
R_{0}=\frac{\tilde{E}}{2}\left(\tilde{E}\left(1-\lambda \varepsilon^{2}\right) \pm \sqrt{\left(1-\lambda \varepsilon^{2}\right)^{2} \tilde{E}^{2}-4 \tilde{L}^{2}}\right) .
$$

Thus when there is no angular momentum we see that the zero of the potential occurs at $R_{0}=0$ and/or $R_{0}=\tilde{E}^{2}\left(1-\lambda \varepsilon^{2}\right)$ as expected. For non-zero angular momentum we again find our constraint condition. Provided the constraint condition is not broken, we see that there will be zeros of the potential and thus bound orbits will exist.

Now we are in a position to discuss the probe motion in the throat. We have already seen that for $\tilde{L}=0$, the probe either passes through the fivebranes or is bound to 
some maximum distance. This is modified somewhat with the addition of the angular momentum term. We now find that;

$$
R(t \rightarrow \pi / 2)=\frac{\tilde{E}^{2} \bar{k}\left(1-\lambda \varepsilon^{2}\right) \pm \sqrt{\tilde{E}^{4} \bar{k}^{2}\left(1-\lambda \varepsilon^{2}\right)-4 \tilde{L}^{2}}}{2}
$$

where the angular momentum constraint must be satisfied for the solution to remain real. In this instance, for an outgoing probe, we find the same behaviour as we did for purely radial motion. The probe starts off at some minimum distance and moves outward to a maximum distance which is dependent on the strength of the energy density and the electric field, before returning to the minimum. If we now consider the in-falling brane, we find different behaviour. The probe starts off at some maximum distance and travels down the throat toward the fivebranes. If the energy is sufficiently large the probe will reach $R=0$. If not, then it will be in a bound state with the fivebranes. The exact position of the bound state in the throat will be dependent upon the strength of the electric field.

In the large $R$ limit we see that the effective potential reproduces the purely radial one, since the angular momentum becomes negligible at large distances (in fact the angular momentum is negligible for all distances much larger than the compactification radius as can be seen in figure 3.)

In this case the equation of motion can again be solved explicitly with the solution

$$
R(t)=\frac{\sqrt{t^{2} \tilde{E}^{4}\left(1-\lambda \varepsilon^{2}\right)^{2}-2 \tilde{E}^{2} t^{2}\left(1-\lambda \varepsilon^{2}\right)+t^{2}+\tilde{E}^{2} \tilde{L}^{2}}}{\left(\tilde{E} \sqrt{\left(\tilde{E}^{2}\left(1-\lambda \varepsilon^{2}\right)-1\right)}\right)}
$$

The effect of the electric field in this case will be to alter the position of the turning point, moving it toward zero as the field strength increases.

Using our canonical momenta we can find the equation of motion for the angle $\theta$ in terms of a fixed energy density

$$
\dot{\theta}=\frac{\tilde{L}}{\tilde{E} H R^{2}} .
$$

Far from the throat, where $H \rightarrow 1$, we find that

$$
\dot{\theta}=\frac{\tilde{L}}{\tilde{E} R^{2}}
$$

and upon integration we have

$$
\theta=\frac{\tilde{E}^{2} \arctan (Q t / \tilde{E} \tilde{L})-2 \tilde{E}^{4} \arctan (Q t / \tilde{E} \tilde{L})+\tilde{E}^{6} \arctan (Q t / \tilde{E} \tilde{L})}{Q}
$$


Where we have defined $Q=\sqrt{\tilde{E}^{4}\left(1-\lambda \varepsilon^{2}\right)^{2}-2 \tilde{E}^{2}\left(1-\lambda \varepsilon^{2}\right)+1}$. This shows that the angle initially changes rapidly but then tends toward a fixed value as time evolves. However in the throat we can use our standard approximation of the harmonic function and we find

$$
\dot{\theta}=\frac{\tilde{L}}{\tilde{E} \bar{k} R} .
$$

This can be integrated, but the resulting expression is extremely complicated. We perform a Taylor expansion for small $t$ to give us a description of the early time behaviour, which shows that to leading order the angle changes logarithmically. This is different to the uncompactified case [2], where it was found that the probe spiralled around the throat toward the fivebranes.

If these bound orbits can occur, we want to know what their trajectories are. Fortunately we are able to make some headway with this since the orbit of the probe brane in the near horizon geometry is given by:

$$
\phi-\phi_{0}=\int \frac{d x}{\sqrt{-x^{2}+\tilde{B} x+C}}
$$

Where we have defined $x=1 / R$ as is customary, and also

$$
\begin{gathered}
\tilde{B}=\frac{\tilde{E}^{2} \bar{k}\left(1-\lambda \varepsilon^{2}\right)}{\tilde{L}^{2}} \\
C=\frac{-1}{\tilde{L}^{2}} .
\end{gathered}
$$

This allows us to solve the integral, which up to arbitrary constants, becomes

$$
\tan (\phi)=\frac{x-1 / 2 \tilde{B}}{\sqrt{-x^{2}+\tilde{B} x+C}} .
$$

Upon expansion this yields a simple quadratic equation and so we obtain the explicit solution for the radius of the orbit,

$$
R=2\left(\tilde{B} \pm \sin (\phi) \sqrt{\tilde{B}^{2}+4 C}\right)^{-1} .
$$

The first thing to note is that there will be an orbit of constant radius, ie circular, if

$$
\tilde{L}=\frac{\tilde{E}^{2} \bar{k}\left(1-\lambda \varepsilon^{2}\right)}{2},
$$

which is the solution we obtained from the equations of motion. Provided that the angular momentum constraints are fulfilled, we appear to have elliptic orbits parameterised by

$$
a=\frac{\tilde{L}^{2} \tilde{E}^{2} \bar{k}\left(1-\lambda \varepsilon^{2}\right)}{2}, \quad e=\sqrt{1-\frac{4 \tilde{L}^{2}}{\tilde{E}^{4} \bar{k}^{2}\left(1-\lambda \varepsilon^{2}\right)^{2}}} .
$$


where, as usual, $a$ is the semi-latus rectum, and $e$ is the eccentricity of the orbit. These orbits are just the standard conic sections one would expect in this background.

\subsection{Deep throat region.}

We will now examine what happens as the probe brane reaches a distance that is comparable with the compactification radius. In this instance we must resort to (2.4)

$$
H=1+k l_{s}^{2} \sum_{n=-\infty}^{\infty} \frac{1}{Z^{2}+\left(Y-2 \pi R_{c} n\right)^{2}},
$$

where we have chosen to parameterise the coordinates on the $S^{1}$ by $Y$, and the transverse coordinates in $\mathbf{R}^{3}$ by $Z$ for convenience.

Since this solution will be valid deep in the CHS throat, we choose to rescale the distances by a factor of $g_{s}$, and then send $g_{s} \rightarrow 0$ whilst keeping the rescaled distances fixed - which is the limit taken when discussing Little String Theory [10].

$$
Y=g_{s} y ; \quad Z=g_{s} z ; \quad R_{c}=g_{s} r_{c}
$$

The harmonic function in terms of these rescaled distances is given by

$$
H(z, y)=\frac{k l_{s}^{2}}{2 r_{c}|z|} \frac{\sinh \left(|z| / r_{C}\right)}{\left(\cosh \left(|z| / r_{c}\right)-\cos \left(y / r_{c}\right)\right)}
$$

where $y$ ranges from $0 \ldots \pi r_{c}$. This parameterisation is chosen to ensure that the fivebranes sit at $y=0$. At relatively large transverse distance, we can neglect the $y$ term which implies that the harmonic function becomes

$$
H(z, 0) \approx \frac{k l_{s}^{2}}{2|z| r_{c}} \tanh \left(|z| / 2 r_{c}\right),
$$

whilst at very small distances (or equivalently when the z field is minimized) we find

$$
H(0, y) \approx \frac{k l_{s}^{2}}{4 r_{c}^{2} \sin \left(y / 2 r_{c}\right)^{2}}
$$

The behaviour of a probe brane with this harmonic function was examined in [8], where the relationship between BPS and Non-BPS branes was elucidated. The probe brane appears to be stable if placed at $y=\pi r_{c}$, but this is in fact a point of instability. As a result, the probe will be attracted toward the fivebranes located at $y=0$ or $y=2 \pi r_{c}$. This can be viewed in terms of the rolling tachyon using the tachyon map described in an earlier section. Note that if we send $y \rightarrow 0$ we recover the usual $S O(4)$ symmetry associated with the uncompactified case. 
The action for the probe brane in this background is similar to the one in the earlier sections, except that we have a rescaled tension $T_{p}=g_{s} \tau_{p}$, and we can consider velocity in the $z$ and $y$ directions. The rescaled energy in this region is given by

$$
\tilde{E}=\frac{1}{H(z, y) \sqrt{H^{-1}(z, y)\left(1-\lambda \varepsilon^{2}\right)-\left(\dot{z}^{2}+\dot{y}^{2}\right)}},
$$

and the equations of motion for fixed energy are given by

$$
\dot{z}^{2}+\dot{y}^{2}=\frac{\left(1-\lambda \varepsilon^{2}\right)}{H(z, y)}-\frac{1}{\tilde{E}^{2} H^{2}(z, y)} .
$$

We know that for distances much larger than $r_{c}$ we can use the results from the previous section, whilst for distances smaller than $r_{c}$ we effectively have a dimensionality crossover where the harmonic function behaves as $1 / r^{2}$. Thus, it is useful to investigate what happens when the probe brane is at a distance comparable with the compactification radius.

From the equations of motion, we have the non-negativity constraint

$$
\frac{k l_{s}^{2}\left(1-\lambda \varepsilon^{2}\right)}{2|z| r_{c}} \frac{\sinh \left(|z| / r_{c}\right)}{\left(\cosh \left(|z| / r_{c}\right)-\cos \left(y / r_{c}\right)\right)} \geq \frac{1}{\tilde{E}^{2}} .
$$

If we assume that $z \approx r_{c}$ we can perform a Taylor series expansion which yields:

$$
\frac{k l_{s}^{2}\left(1-\lambda \varepsilon^{2}\right)}{2 z^{2}} \frac{1 / 2\left(e-e^{-1}\right)}{1 / 2\left(e+e^{-1}\right)-\cos \left(y / r_{c}\right)} \geq \frac{1}{\tilde{E}^{2}} .
$$

Since the cosine term is constrained to be between \pm 1 , we find that the constraint can be written

$$
\frac{k l_{s}^{2} \mathcal{O}(1)}{2 z^{2}} \geq \frac{1}{\tilde{E}^{2}}
$$

As before we write the effective potential as

$$
V_{\mathrm{eff}}=\frac{1}{\tilde{E}^{2} H^{2}(z, y)}-\frac{\left(1-\lambda \varepsilon^{2}\right)}{H(z, y)}
$$

which we plot in figures 5 and 6 . As we can see, in the region of validity we find that the potential is repulsive for small values of $\tilde{E}$. The minimum potential in this region is at the point $y=\pi r_{c}, z=0$ as expected from [8]. The effect of the electric field in this instance is to reduce the height of the potential along the $\mathbf{S}^{1}$ direction.

\section{Discussion.}

We have seen that by compactifying one of the transverse dimensions to the NS5-brane, we do in fact find bound orbits at sufficiently large distances. The inclusion of an electric 
field on the probe brane also has a strong effect on the dynamics, since we have found that an increasing electric field strength tends to localise the probe brane nearer the fivebranes - i.e in a region of strong coupling. This is what we would expect, since the field is effectively adding more 'mass' to the brane and so we would expect it to sink further into the throat.

Using the tachyon map we have explored the differences between the solutions in a compactified background and the general background, and also seen how they can be mapped to one another. Interestingly we find that there is a unique size of the compactification radius, namely $R_{c}=\sqrt{k l_{s}^{2}} / 2$, which will map the compactified solution to the uncompactified one as $T \rightarrow-\infty$. It would be interesting to understand the underlying reasons for this.

When we include the angular momentum term we expect to see different dynamics. However there are constraints emerging from the throat solution which suggest that the angular momentum term is negligible in the near horizon region. If the angular momentum saturates the equation of motion constraint, then we will find that the probe executes circular motion in the throat at a distance determined by the compactification radius and the electric field strength. Since the $D$-brane will constantly be radiating energy as it moves, this trajectory is unlikely to be stable - however since the probe carries RamondRamond charge there may be something akin to synchrotron radiation emission [27]. The dynamics of the probe as it approaches the compactification radius have also been addressed.

In closing it appears that we have verified Kutasov's conjecture and also seen the effect that an electric field has on the probe dynamics. There is still the issue of the critical angular momentum to consider, since altering the background geometry appears to have no effect on this constraint. Further work must be done to determine the underlying reasons for this. The work could also be extended to consider $D p$-brane backgrounds and also other types of intersecting brane backgrounds with relative ease. Also the presence of a bound state could be useful for cosmology, in particular an extension of the work already done in [3], [16] and [11]. In relation to the work done in [8], it would also be of interest to consider Non-BPS branes in this background. Recent work on a related problem has appeared in [18], [20].

Added Note: After completing this work we became aware of ref [26] which has a sizable overlap with the present paper. However we have in addition, also presented in our work, analytic solutions regarding the probe brane orbits. 


\section{References}

[1] C. G. Callan J. A. Harvey and A. Strominger, 'Supersymmetric sting solitons', hep-th/9112030, K. S. Stelle, 'BPS branes in supergravity, hep-th/9803116.

[2] David Kutasov, 'D-brane dynamics near NS5-branes', hep-th/0405058.

[3] A. Kehagias and E. Kiritsis, 'Mirage Cosmology', hep-th/9910174, JHEP 9911 (1999) 022 .

[4] Amanda W. Peet, 'Black holes in string theory', hep-th/0008241.

[5] C. P. Burgess, P. Martineau, F. Quevedo and R. Rabadan, 'Branonium', hep-th/0303170, JHEP 0306 (2003) 037.

[6] C.P. Burgess, P. Martineau, F. Quevedo and R. Rabadan, 'D-brane Chemisty', hepth/031001, JHEP 0401 (2004) 067.

[7] K. L. Panigrahi, 'D-Brane dynamics in Dp-background', hep-th/0407134, Phys.Lett. B601 (2004) 64-72.

[8] David Kutasov, 'A geometric interpretation of the open string tachyon', hep-th/0408073.

[9] David. A. Sahakyan, 'Comments on D-brane Dynamics Near NS5-Branes', hep-th/0408070, JHEP 0410 (2004) 008.

[10] O. Aharony, M. Berkooz, D. Kutasov and N. Seiberg, 'Linear dilatons, NS5-branes and holography', hep-th/9808149, JHEP 9810 (1998) 004.

[11] Ahmad Ghodsi and Amir E. Mosaffa, 'D-brane Dynamics in RR Deformation of NS5-branes Background and Tachyon Cosmology', hep-th/0408015.

[12] G. W. Gibbons, 'Cosmological Evolution of the Rolling Tachyon', hep-th/0204008 Phys.Lett. B537 (2002) 1-4.

[13] A. Sen, 'Tachyon matter', hep-th/0203265, JHEP 0207 (2002) 065.

[14] A. Sen, 'Field theory of Tachyon matter', hep-th/0204143, Mod.Phys.Lett. A17 (2002) 1797-1804.

[15] C. V. Johnson 'D-branes', Cambridge University Press 2003.

[16] Hossein Yavartanoo, 'Cosmological Solution from D-brane motion in NS5-Branes background', hep-th/0407079.

[17] M.R. Garousi 'Tachyon couplings on non-BPS D-branes and Dirac-Born-Infeld action', hep-th/0003122, Nucl.Phys. B584 (2000) 284-299 
[18] J. Kluson, 'Non-BPS D-brane Near NS5-branes', hep-th/0409298, JHEP 0411 (2004) 013.

[19] J. Kluson, 'Non-BPS Dp-Brane in the Background of NS5-Branes on Transverse $\mathbf{R}^{3} \times S^{1}$, hep-th/0411014.

[20] J. Kluson, 'Non-BPS Dp-Brane in Dk-Brane Background', hep-th/0501010.

[21] Dongsu Bak, Soo-Jong Rey and Ho-Ung Yee, 'Exactly Soluble Dynamics of (p,q) String Near Macroscopic Fundamental Strings', hep-th/0411099, JHEP 0412 (2004) 008.

[22] Steven Thomas and John Ward, 'D-Brane Dynamics and NS5 Rings', hep-th/0411130, JHEP to appear.

[23] Y. Nakayama, Y. Sugawara and H. Takayanagi, 'Boundary states for the rolling D-branes in NS5-background', hep-th/0406173, JHEP 0407 (2004) 020.

[24] Y. Nakayama, K. Panigrahi, S. J. Rey and H. Takayanagi, 'Rolling Down the Throat in NS5-Brane Background: The case of Electrified D-Brane', hep-th/0412038.

[25] B. Chen, M. Li and B. Sun, 'Dbrane Near NS5-branes: with Electromagnetic Field', hep-th/0412022.

[26] B. CHen and B. Sun, 'Note on DBI dynamics of Dbrane Near NS5-branes', hep-th/0501176.

[27] Troels Harmark and Konstantin G. Savvidy, 'Ramond-Ramond Field Radiation from Rotating Ellipsoidal Membranes', hep-th/0002157, Nucl.Phys. B585 (2000) 567-588. 


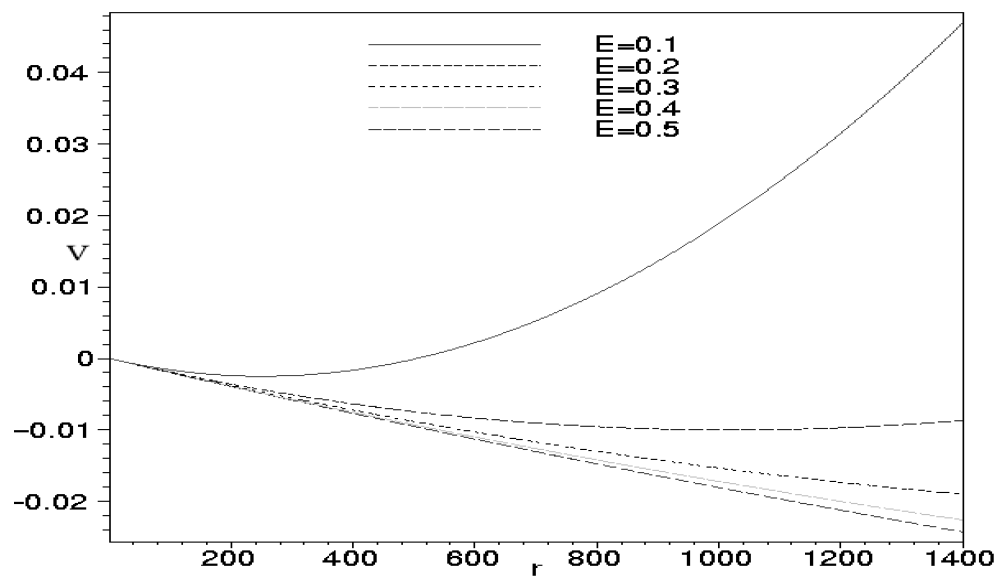

Figure 1: Potential for various energy density values with zero field strength. We have taken $k=100000, l_{s}=1$ and $R_{c}=1$ for simplicity.

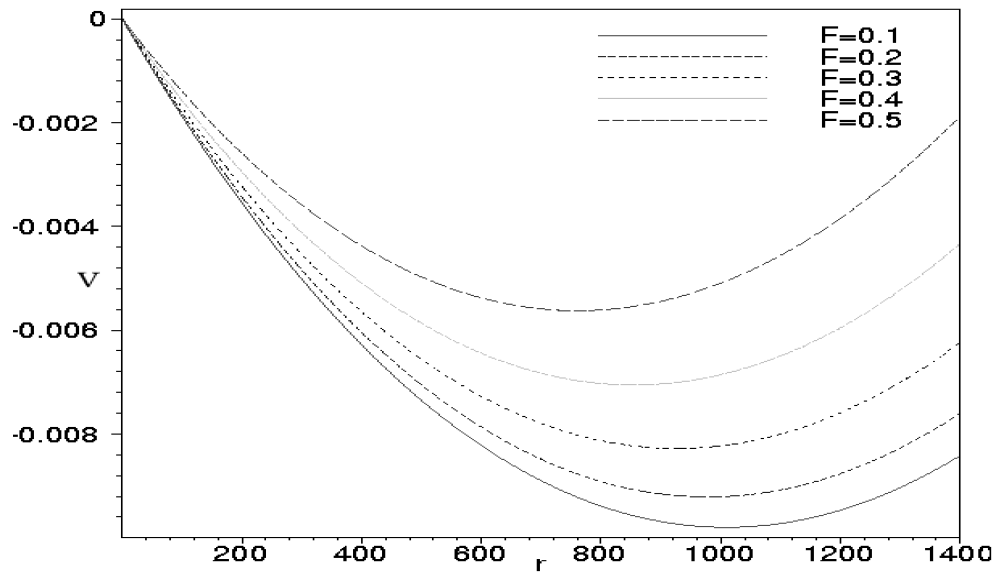

Figure 2: Potential for fixed $\tilde{E}<<1$ but with increasing (dimensionless) electric field strength. Note that the stronger electric field shifts the minimum toward $r=0$. 


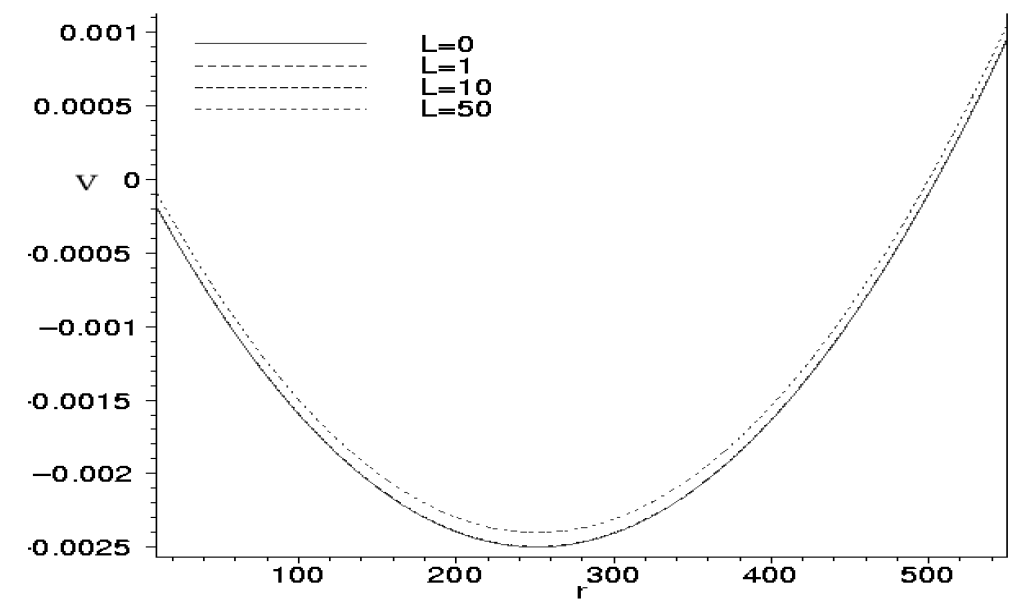

Figure 3: Potential for fixed $\tilde{E}<<1$, and zero electric flux. The angular momentum appears to be negligible in this region.

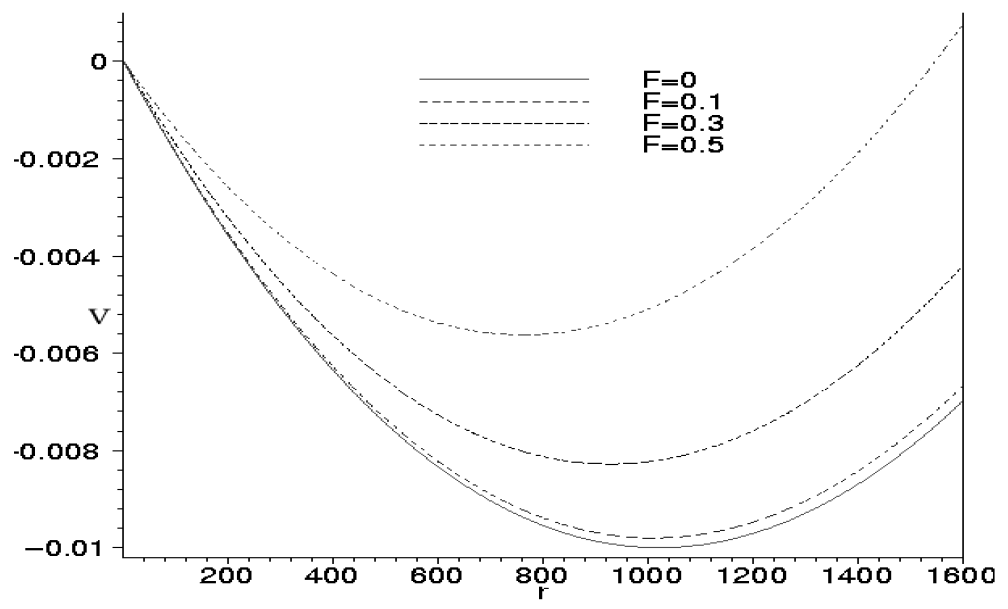

Figure 4: Potential with $\tilde{L}=1$ and fixed energy density, $\tilde{E}<<1$. We see that the effect of the electric field is to shift the position of the minima to smaller $r$. 


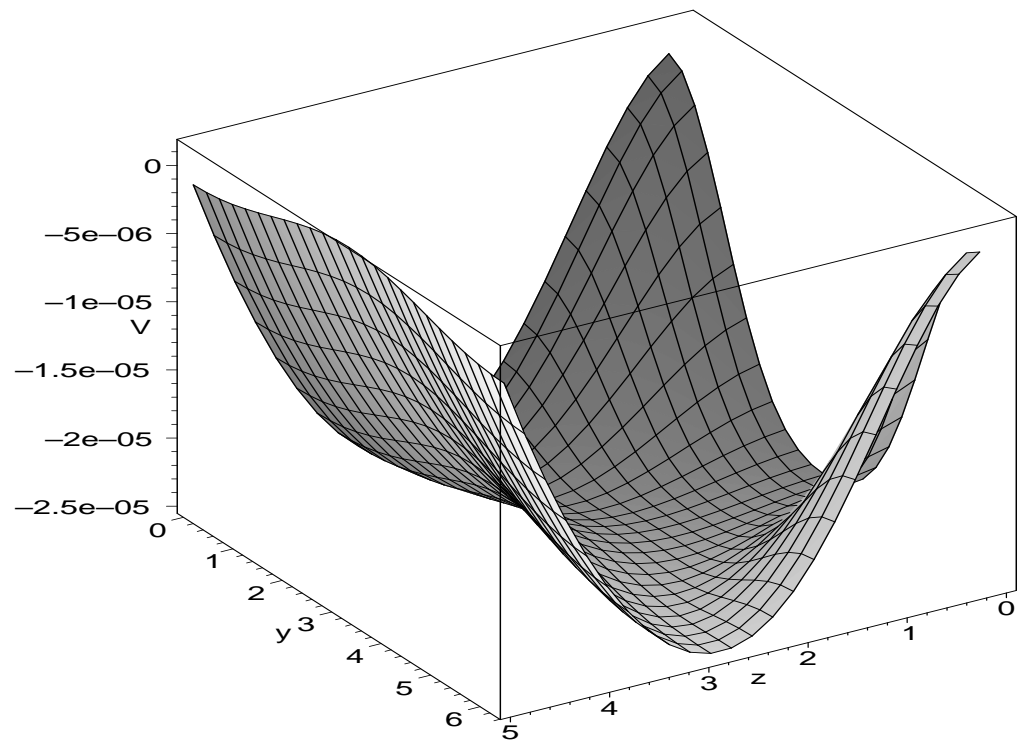

Figure 5: Potential for $\tilde{E}=0.01$ as the probe moves into a region where it is comparable with the compactification radius. We have set the electric field to zero here.

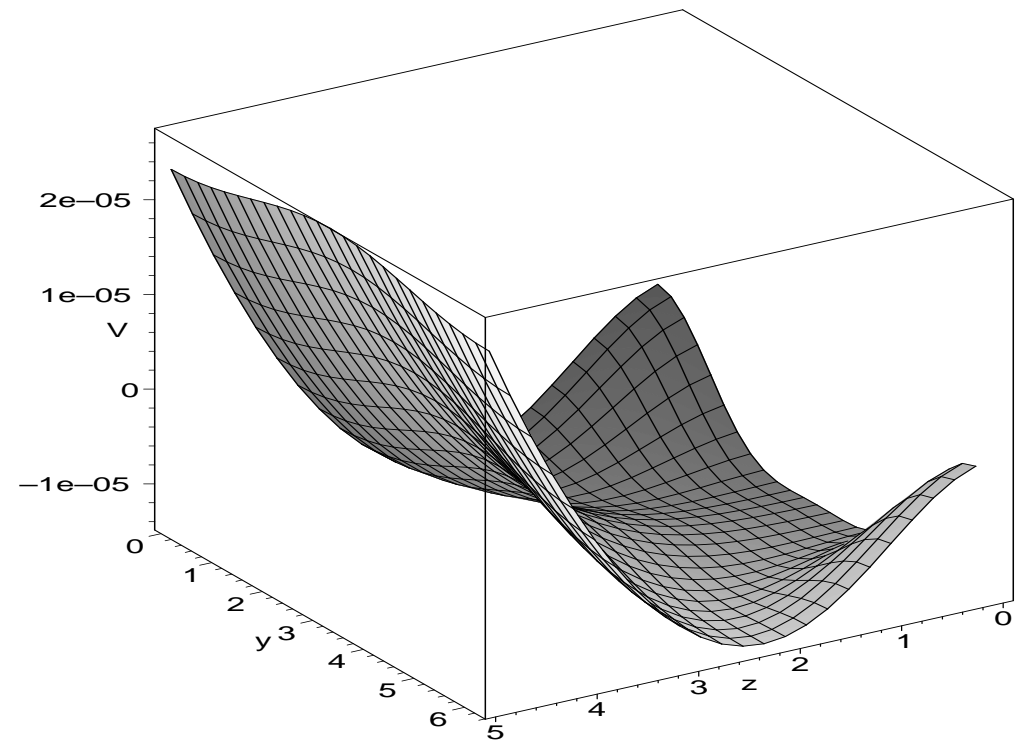

Figure 6: Potential for $\tilde{E}=0.01$ with an electric field strength of $\mathrm{F}=0.5$ showing how the electric field alters the potential along the circle when $\mathrm{z}=0$. 